\title{
Rare Types of Rotaviruses Isolated from Children with Acute Gastroenteritis in Patras, Greece
}

\author{
Petros A. Kokkinos ${ }^{a}$ Panos G. Ziros ${ }^{a}$ Marina Monini ${ }^{c}$ Petroula Lampropoulou ${ }^{a}$ \\ Anthoula Karampini $^{b}$ Eleni Papachatzi ${ }^{a}$ Stefanos Mantagos ${ }^{b}$ \\ Franco M. Ruggeric Apostolos Vantarakis ${ }^{\mathrm{a}}$ \\ ${ }^{a}$ Environmental Microbiology Unit, Department of Public Health, School of Medicine, University of Patras, and \\ ${ }^{b}$ Department of Pediatrics, University Hospital of Patras, Patras, Greece; ${ }^{\mathrm{c}}$ Department of Veterinary Public Health \\ and Food Safety, Istituto Superiore di Sanità, Rome, Italy
}

\section{Key Words}

Pediatric gastroenteritis · Molecular epidemiology ·

Rotavirus · Enterovirus · Adenovirus · Emerging viruses

\begin{abstract}
Background: Acute diarrhea remains a major public health issue worldwide, with gastroenteritis agents associated with a high morbidity, especially in infants and young children. A 1-year study (2009-2010) of rotavirus (RV)-caused pediatric diarrhea was performed on hospitalized children admitted with symptoms of acute diarrhea to the University Hospital of Patras, Greece. Methods: Twenty-nine fecal samples were investigated for RVs, adenoviruses (AdVs), and enteroviruses (EVs) in an attempt to characterize these enteric viruses, which have been implicated in hospitalized diarrhea. $R \boldsymbol{e}$ sults: A 44.8\% (13/29) incidence of viral infection was reported for the viral targets. Monoinfections accounted for $31 \%$ (9/29), while bi-infections accounted for $13.4 \%$ (4/29). Sequencing of positive samples allowed identification of RVs G4P[8] (2/29), G9P[8] (1/29), and interestingly of the rare type G12P[8] (2/29). AdV types 1, 2, and 6 were also identified in $4 / 29,1 / 29$, and $1 / 29$ of the samples, respectively. Regarding the EVs, EV71 (2/29), coxsackievirus A4 (1/29), echovirus 11
\end{abstract}

\section{KARGER}

E-Mail karger@karger.com

www.karger.com/int
(1/29), and EV96 (1/29) were typed. Conclusion: The results of the present study, and specifically the detection of rare RV G12 and EV71 strains, address the need for continuous epidemiological surveys to provide epidemiological pictures of pediatric viral infections circulating in the community.

Copyright $\odot 2013$ S. Karger AG, Basel

Gastroenteritis agents are associated with a high morbidity, especially in infants and young children, and cause large outbreaks and high numbers of sporadic cases worldwide. Known and emerging enteric viruses are recognized as important pathogenic agents [1]. Nevertheless, the molecular epidemiological characteristics of infant and pediatric gastroenteritis associated with viral agents are poor in many countries, mainly due to the inexistence of specific national surveillance systems for gastroenteritis. Only limited data on enteropathogenic viruses are available from Greece, where there is no systematic surveillance system for viral gastroenteritis [2]. Rotaviruses (RVs) are enteric pathogens in humans and animals that are found worldwide. Group A RVs are the most important causative agents of severe childhood diarrhea. RV gastroenteritis represents a major public

Dr. Apostolos Vantarakis

Environmental Microbiology Unit, Department of Public Health School of Medicine, University of Patras

GR-26504 Rion (Greece)

E-Mail avanta@upatras.gr 
health burden in the EU and is responsible for almost half of the acute gastroenteritis (AGE) hospitalizations and emergency visits in children up to 5 years of age in Greece $[3,4]$. RV genotype distribution varies seasonally and among countries and regions. Studies from European centers report G1P[8], G2P[4], G9P[8], G3P[8], and $\mathrm{G} 4 \mathrm{P}[8]$ as their most frequently identified genotypes [3]. A growing number of countries worldwide have reported the occurrence of G12, both sporadically and as a genotype of notable incidence [5-9]. To the best of our knowledge, the G12 genotype has not previously been reported in Greece.

Human adenoviruses (AdVs) are divided into six subgroups (A-F) comprised of 51 different serotypes [10]. Out of the six subgroups, AdVs of subgroup F (enteric serotypes 40 and 41) are estimated to be associated with 5-20\% of AGE cases among infants and young children [2]. Human enteroviruses (EVs) belong to the family Picornaviridae and are among the most common causes of acute viral illness in childhood [11]. A recent study described the identification of strains belonging to human $\mathrm{EV}$ species B and A in children and the detection of the emerging pathogen EV71 for the first time in Greece [12]. To evaluate the epidemiological and clinical role of different viruses in sporadic pediatric gastroenteritis patients in Italy, a 1-year study (2008-2009) showed that RV was the pathogen most frequently detected (38.3\%), with AdVs detected at $2.6 \%$ and EVs not detected at all [1].

The objective of the present study was to evaluate the incidence of RV, AdV, and EV in hospitalized children with diarrhea at the University Hospital of Patras in southwestern Greece during the period 2009-2010. During the 1-year study period (March 2009 to March 2010), stool specimens were collected from 29 of the hospitalized children suffering from nonbacterial AGE who agreed to participate in the study. Demographic (age, sex, residence, school, specific population, and vaccination) and clinical data such as days of hospitalization and symptoms (vomiting, mucus, diarrhea, bloody stools, fever, and abdominal pain) were collected from self-administered questionnaires. During the study period, no AGE outbreak was reported. Stool samples were collected in sterile containers and transported immediately to the laboratory. They were collected within the first $24 \mathrm{~h}$ of hospitalization to avoid the possibility of nosocomial viral infection. Samples were initially screened for bacterial pathogens (Salmonella spp., Shigella, Escherichia coli, Pseudomonas spp.) and stored at $-80^{\circ}$ as a $10 \%$ fecal suspension in a balanced salt solution until they were processed for virological analysis. Viral nucleic acids were extracted from stool specimens using the QIAamp Viral RNA mini-kit (Qiagen, Hilden, Germany), using the QIAcube fully automated platform. PCR, RT-PCR, and nested PCR techniques were used for the detection of the selected viral targets, according to previously published protocols [13-17], by targeting the hexon gene (AdVs), gene 9 (RVs), and $5^{\prime}$ NTR (EVs). Extracted RV dsRNA was first reverse-transcribed into gene 9 full-length cDNA with the generic primers Beg9/End9 (1,062 bp) [16]. Then, in a second step, the cDNA product was used as a template for PCR VP7 amplification with the same Beg9/ End9 pair of primers [14]. For P-typing, primers Con2Con3 [17], or primers VP4F/VP4R [13], were used for amplification of a VP4-specific region. For AdV DNA amplification, primers HexA/HexB (308 bp) and nHexA/ nHexB (143 bp) were used, while for EV RNA amplification, Ent $1 /$ Ent2 (540 bp) and nEnt1/nEnt2 (123 bp) primers were used. Reverse transcription and first PCR amplification for RV and EV were performed in a one-tube format with the Qiagen one-step RT-PCR (Qiagen). Positive PCR products were purified using the QIAquick PCR purification kit (Qiagen, USA) and QIAcube, and confirmed by sequencing (Sequencing Unit, School of Medicine, University of Thessaly, Thessaly, Greece). Positive G12 RV strains were also sent to Istituto Superiore di Sanità (ISS, Rome, Italy) for confirmation analysis. The obtained nucleotide sequences were analyzed by the BLAST program at the NIH website (NCBI, National Center for Technology Control, NIH, USA), and were compared with each other and with other published sequences. Multiple alignments were performed using the MEGA Molecular Evolutionary Genetics Analysis version 4.0.2 program. The neighbor-joining method was applied for the phylogenetic tree analysis, the reliability of which was assessed by bootstrap resampling $(1,000$ pseudoreplicates) using the MEGA program.

The median age of all children with gastroenteritis was 24 months (range: 1-120). The average age of the hospitalized children was 16.5 months. Ninety-eight of the admitted children were less than 12 months (23.5\%). The children mainly lived in three prefectures of western Greece (Aitoloakarnania, Achaia, Ilia), which make up the fourth most populated region in Greece with $7 \%$ of the country's total population (741,282 inhabitants, 2011 census). $56.4 \%$ of the admitted children were male. $16.3 \%$ of the total admitted children were immigrants or Roma. The symptoms of the patients from which the samples derived were vomiting, mucus, diarrhea, bloody stools, fever, and abdominal pain, and were reported in $41.37 \%$ (12/29), 41.37\% (12/29), 96.5\% (28/29), 44.82\% (13/29), 
Fig. 1. Neighbor-joining phylogenetic dendrogram representing phylogenetic relationships among the two Greek RVG12 strains (highlighted with arrows), and 22 reference strains, the sequences of which were retrieved from the GenBank database. The strain name is followed by the viral host ( $\mathrm{Hu}=$ human; Po = porcine), as well as the country of origin. G12 lineages I, II, III, IV are presented on the right. GenBank accession Nos. of VP7 genes include G12-I (EF672595.1 Hu/L26 Philippines G12-I), G12-II (AB186120.1 Hu/K12 Japan, AB071404.1Hu/T152Thailand,EU496259.1 $\mathrm{Hu} /$ Kor588 South Korea, EU496257.1 Hu/ Arg720 Argentina), G12-III (DQ146665.1 $\mathrm{Hu}$ /Dhaka12-03 Bangladesh, AB264004.1 $\mathrm{Hu} / 04 \mathrm{~S} 010 \mathrm{Nepal}$, EU839935.1 Hu/MMC29 Bangladesh, DQ146687.1 Hu/N26-02 Bangladesh, AB263989.1 Hu/N158 Nepal, AY206861.1 Hu/ISO 1 India, FJ747618.1 Hu/GER126-08 Germany, DQ490550.1 Hu/ RV161-00 Bangladesh, Q017650.1 Hu/ ISO14 India, AJ311741.1 Hu/Se585 USA, DQ062130.1 Hu/ISO30 India, FJ152110.1 $\mathrm{Hu} / \mathrm{US} 6588$ USA, EF059917.1 Hu/CAU 214 South Korea, AB269689.1 Hu/MD844 Saudi Arabia, DQ146643.1 Hu/B4633-03 Belgium, DQ146676.1 Hu/Matlab13-03 Bangladesh), and G12-IV (DQ204743.1 Po/ RU172 India).

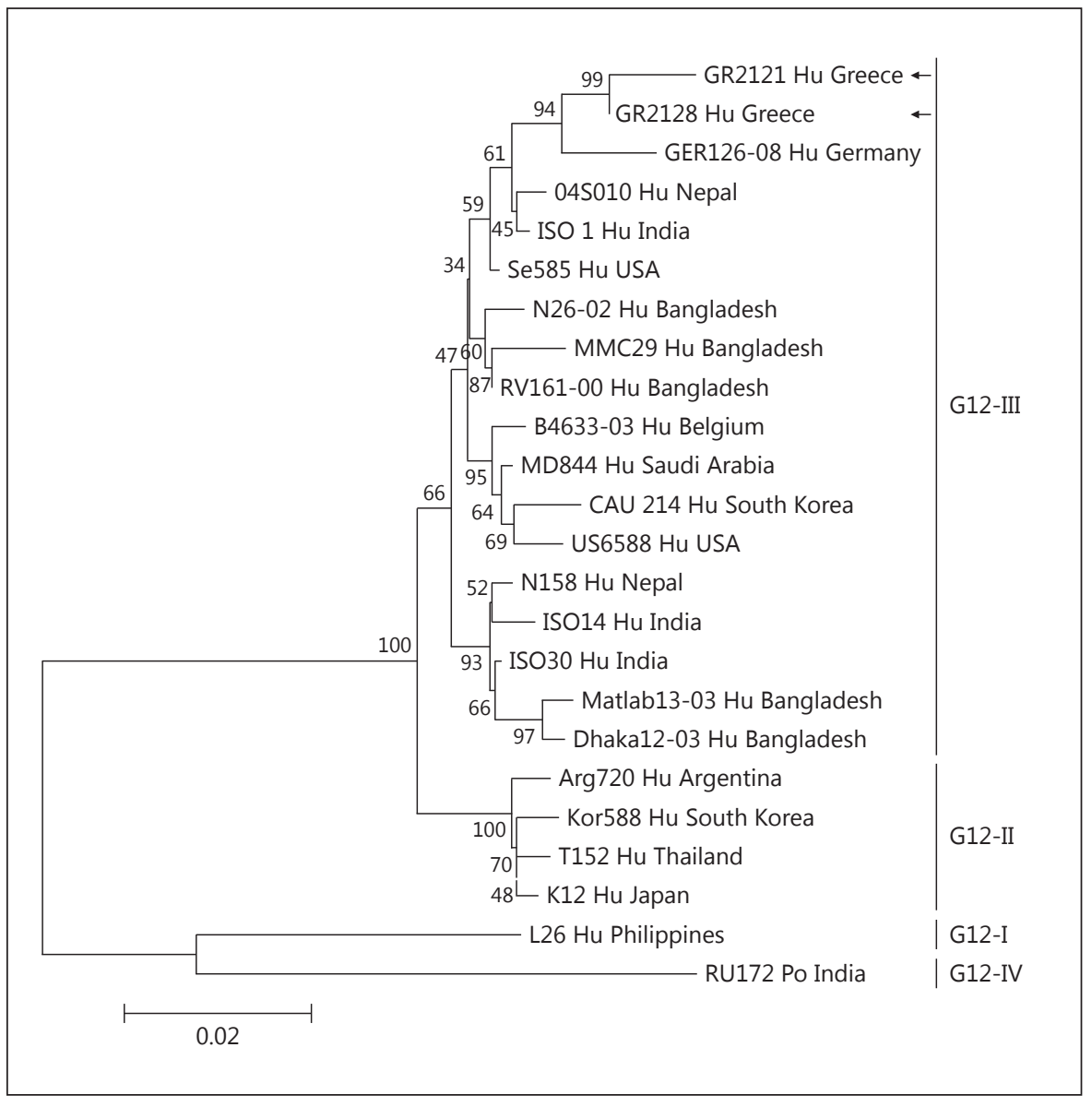

$58.62 \%(17 / 29)$, and $10.34 \%(3 / 29)$ of the patients, respectively. Roma accounted for $20.68 \%$ (6/29), while immigrants accounted for $10.34 \%(3 / 29)$. The median age of the patients with positive fecal samples for the tested viral targets was 16.4 months; the age range was 4-30 months, 4 patients were aged less than 12 months, and there were 9 males and 4 females. Only 2 samples were also found to be positive for a bacterial pathogen [Pseudomonas spp. $(\mathrm{n}=1)$, Salmonella enterica serovar enteritidis $(\mathrm{n}=1)$, multisensitive]. No target viruses were detected in the Salmonella-positive sample, while the Pseudomonas-positive sample was positive for both $\mathrm{AdV}$ (AdV1) and EV (EV96). Of the 29 stool samples collected, 5 were obtained during autumn, 14 during winter, 5 during spring, and 5 during summer. In total, viruses were detected in $44.8 \%(13 / 29)$ of the analyzed fecal samples. Viral monoinfections accounted for 31.0\% (9/29), while viral bi-infections accounted for $13.4 \%(4 / 29)$. In detail, AdV and EV were found to be simultaneously present in 2 samples, while EV and RV were both detect- ed in the other 2 samples. AdV types 1,2, and 6 were recognized in $13.8 \%(4 / 29), 3.4 \%(1 / 29)$, and $3.4 \%(1 / 29)$ of the patients, respectively. Regarding the EVs, EV71 (2/29), coxsackievirus A4 (1/29), echovirus $11(1 / 29)$, and EV $96(1 / 29)$ were identified. RVs G4P[8] (2/30), G9P [8] (1/30), and G12P[8] (2/30) were detected. Positive RVG12P[8] strains were found in specimens from a 72-month-old male and a 30-month-old female, both Roma from Ilia. These 2 patients were hospitalized in December 2009 and January 2010, respectively. The boy had a negative stool culture for bacterial pathogens and presented with vomiting, diarrhea, fever, and abdominal pain, but no bloody stools or mucus, while the girl presented with vomiting, mucus, diarrhea, fever, and no bloody stools or abdominal pain. The two children had not previously been vaccinated against RVs. The phylogenetic analysis is summarized in figure 1.

A neighbor-joining phylogenetic tree was constructed to illustrate the phylogenetic relationships among the two Greek RVG12 strains and 22 reference strains (fig. 1) 
which represent lineages I-IV. Greek RVG12 strains clustered with a German strain (GER126-08) and belonged to G12-III lineage. A nucleotide alignment to compare the two Greek RVG12 strains to each other showed a very high nucleotide identity of 99\%. Both Greek strains showed a nucleotide identity of $98 \%$ to the German strain GER126-08 (GenBank accession No. FJ747618). VP7 nucleotide alignments of the two Greek strains with published sequences showed a 99\% homology to G12-III strains CU607-TK/09 (Thailand), ISO129 (India), and KUH146 (Sri Lanka). Isolated EV strains belonged to species EV-A $(\mathrm{n}=3)$, EV-B $(\mathrm{n}=1)$, and EV-C $(\mathrm{n}=1)$. GREV1914 isolate was identified as an EV71 strain, and presented a 100\% nucleotide identity to isolates EV054_07 (Canada), 10118 (the Netherlands), NUH0075/SIN/08 (Singapore), ENT/PM/SHA71 (Malaysia), and HME-270 (Taiwan). GREV 1917 had a 96\% nucleotide identity with human EV96 strain BAN00-10488 (Bangladesh), while GREV1923 strain had a 91\% nucleotide identity to EV71 isolates THA-EV71-002 (Thailand), 15-AUT2001 (Austria), and NSW/182/98 (Australia). Finally, GREV2128 presented a $100 \%$ nucleotide identity to human echovirus 11 isolate India115 (India) and isolate NSW/19/97 (Australia). Finally, GREV2130 strain had an 88\% homology to human coxsackievirus A4 strain High Point.

$\mathrm{RV}$ is the most common cause of AGE in children up to 5 years of age in Greece. It has a greater clinical burden compared to non-RV AGE, and it occurs more frequently in winter months [3]. Five common serotypes (G1, G2, G3, G4, and G9) are responsible for over $98 \%$ of all RV infections in Europe [4]. Studies on RV genotype distribution are very few in Greece, although some remarkable works have recently been published $[3,18]$. During a multicenter study conducted during the period 20072008, a strain that displayed the unusual G12P[14] type was finally identified as G8P[14] [3]. To the best of our knowledge, RVG12 strains were never reported previously in Greece. In the present study, RV G4P[8] (2/29), G9P [8] (1/29), and G12P[8] (2/29) were detected, mainly during winter (4/5 during winter, $1 / 5$ during March 2010).

It is possible that collecting data from only one center might affect the genotyping analysis since genotype distribution may significantly vary in different regions. A further limitation in this study is due to the low number of analyzed samples. This occurred due to the refusal of patients to participate in the study. Nevertheless, the detection of two positive RVG12 strains in the analyzed samples is suggestive of some circulation of this uncommon viral genotype at least in the southwest of Greece, and underlines the need for further investigations on any changes in the genotype distribution and the emergence of unusual genotypes in order to provide a true epidemiological picture. Although RV vaccines have been on the market in Greece for the last 5 years and can be administered by local pediatricians, the vaccine has only recently been incorporated into the Greek NIP, and fulldose vaccination coverage has remained lower than $30 \%$ (at least until 2009-2010) [4, 18].

Although enteric AdV types 40 and 41 seem to predominate, nonenteric subgenera (A and $\mathrm{C}$ ) can also be causally implicated [2]. In our study, AdV types 40 and 41 were surprisingly not detected at all, whereas AdV types 1,2 , and 6 were recognized in $13.3 \%(4 / 30), 3.3 \%$ $(1 / 30)$, and $3.3 \%(1 / 30)$, respectively. This apparent difference might again be due to either geographic or temporal differences or to the limited number of patients investigated presently. In the present study, EV71, coxsackievirus A4, echovirus 11, and EV96 were identified in a total of 5 cases out of 29 tested. EV71 (species human EV-A) appears to be an emerging neurotropic EV since several outbreaks and severe clinical complications with EV71 infection were found $[19,20]$. EV71 infections are common in children and are acquired largely in the (pre) school years, while spread of these viruses is lower in the other age groups [21]. Echovirus 11 (species human EVB) is among the most frequently isolated EV serotypes worldwide, while coxsackievirus A strains, including A4, have been implicated in important epidemics around the world [22]. A recent investigation of EV circulation in children identified strains of EV-B and EV-A. All reported EV-A serotypes of that study, were detected for the first time in Greece, and - most notably- six of them were identified as EV71 [12].

The investigation of VP1-target sequences could have been more useful for the precise description of the molecular epidemiology of the isolated EV strains in the present study. Investigation of other viruses, such as noroviruses, parechoviruses, toroviruses, Aichi viruses, picobirnaviruses, and picotrinavirus might help increase the diagnosis rate in children with gastroenteritis $[1,2]$.

This study was designed to assess the prevalence of selected viral agents in sporadic pediatric gastroenteritis patients attending the University Hospital of Patras and to improve knowledge in the geographical context of western Greece. The study detected the emerging RVG12 strains for the first time in Greece. This finding together with the identification of EV71 strains in a relatively limited number of patients during a 1-year period and from one hospital, emphasizes the need for extended surveil- 
lance studies, especially in pediatric populations, in order to investigate any changes in the genotype distribution with time and the emergence of unusual genotypes of viral enteropathogens.

\section{Disclosure Statement}

There are no sources of funding or competing interests to disclose.

\section{References}

1 Rimoldi SG, Stefani F, Pagani C, Chenal LL, Zanchetta N, Di Bartolo I, Lombardi A, Ruggeri FM, Di Lillo D, Zuccotti GV, Gismondo MR: Epidemiological and clinical characteristics of pediatric gastroenteritis associated with new viral agents. Arch Virol 2011;156: 1583-1589.

- 2 Levidiotou S, Gartzonika C, Papaventsis D, Christaki C, Priavali E, Zotos N, Kapsali E, Vrioni G: Viral agents of acute gastroenteritis in hospitalized children in Greece. Clin Microbiol Infect 2009;15:596-598.

-3 Koukou D, Grivea I, Roma E, Tsioni H, Trimis G, Galanakis E, et al: Frequency clinical characteristics and genotypes distribution of rotavirus gastroenteritis in Greece (20072008). J Med Virol 2011;83:165-169.

-4 Syriopoulou V, Kafetzis D, Theodoridou M, Syrogiannopoulos GA, Mantagos S, Trimis G, et al: Evaluation of the potential medical and economic benefits of universal rotavirus vaccination programme in Greece. Acta Paediatr 2011;100:732-739.

5 Aladin F, Nawaz S, Iturriza-Gómara M, Gray $\mathrm{J}$ : Identification of G8 rotavirus strains determined as G12 by rotavirus genotyping PCR: updating the current genotyping methods. J Clin Virol 2010;47:340-344.

6 Castello AA, Nakagomi T, Nakagomi O, Jiang B, Kang JO, Glass RI, Glikmann G, Gentsch JR: Characterization of genotype P[9]G12 rotavirus strains from Argentina: high similarity with Japanese and Korean G12 strains. J Med Virol 2009;81:371-381.

7 Pietsch C, Liebert UG: Human infection with G12 rotaviruses, Germany. Emerg Infect Dis 2009;15:1512-1515.

8 Cunliffe NA, Ngwira BM, Dove W, Nakagomi O, Nakagomi T, Perez A, Hart CA, Kazembe PN, Mwansambo CC: Serotype g12 rotaviruses, Lilongwe, Malawi. Emerg Infect Dis 2009; 15:87-90.
-9 Oluwatoyin Japhet M, Adeyemi Adesina O, Famurewa O, Svensson L, Nordgren J: Molecular epidemiology of rotavirus and norovirus in Ile-Ife, Nigeria: high prevalence of $\mathrm{G} 12 \mathrm{P}[8]$ rotavirus strains and detection of a rare norovirus genotype. J Med Virol 2012;84: 1489-1496.

10 Haramoto E, Katayama H, Oguma K, Ohgaki $S$ : Quantitative analysis of human enteric adenoviruses in aquatic environments. J Appl Microbiol 2007;103:2153-2159.

11 Dumaidi K, Frantzidou F, Papa A, Diza E, Antoniadis A: Enterovirus meningitis in Greece from 2003-2005: diagnosis, CSF laboratory findings, and clinical manifestations. J Clin Lab Anal 2006;20:177-183.

12 Siafakas N, Attilakos A, Vourli S, Stefos E, Meletiadis J, Nikolaidou P, Zerva L: Molecular detection and identification of enteroviruses in children admitted to a university hospital in Greece. Mol Cell Probes 2011;25:249254.

13 Iturriza-Gomara M, Green J, Brown DW, Desselberger U, Gray JJ: Diversity within the VP4 gene of rotavirus $\mathrm{P}[8]$ strains: implications for reverse transcription-PCR genotyping. J Clin Microbiol 2000;38:898-901.

14 Barril PA, Giordano MO, Isa MB, Masachessi G, Ferreyra LJ, Castello AA, Glikmann G, Nates SV: Correlation between rotavirus A genotypes detected in hospitalized children and sewage samples in 2006, Córdoba, Argentina. J Med Virol 2010;82:1277-1281.

15 Kokkinos P, Filippidou S, Karlou K, Vantarakis A: Molecular typing of enteroviruses, adenoviruses, and hepatitis A viruses in untreated and treated sewage of a biological treatment plant in Greece. Food Environ Virol 2010;2:89-96.
16 Gouvea V, Glass R, Woods P, Taniguchi K, Clark H, Forrester B, Fang Z: Polymerase chain reaction amplification and typing of rotavirus nucleic acid from stool specimens. J Clin Microbiol 1990;28:276-282.

17 Gentsch JR, Glass RI, Woods P, Gouvea V, Gorziglia M, Flores J, Das BK, Bhan MK: Identification of group A rotavirus gene 4 types by polymerase chain reaction. J Clin Microbiol 1992;30:1365-1373.

18 Trimis G, Koutsoumbari I, Kottaridi C, Palaiologou N, Assimakopoulou E, Spathis A, Lebessi E, Konstantopoulos A, Kafetzis D, Karakitsos P, Papaevangelou V: Hospitalbased surveillance of rotavirus gastroenteritis in the era of limited vaccine uptake through the private sector. Vaccine 2011;29:72927295.

19 Tan X, Huang X, Zhu S, Chen H, Yu Q, Wang $\mathrm{H}$, Huo X, Zhou J, Wu Y, Yan D, Zhang Y, Wang D, Cui A, An H, Xu W: The persistent circulation of enterovirus 71 in People's Republic of China: causing emerging nationwide epidemics since 2008. PLoS One 2011; 6:e25662.

20 Ang LW, Phoon MC, Wu Y, Cutter J, James L, Chow VT: The changing seroepidemiology of enterovirus 71 infection among children and adolescents in Singapore. BMC Infect Dis 2011;11:270.

21 Kapusinszky B, Szomor KN, Farkas A, Takács M, Berencsi G: Detection of non-polio enteroviruses in Hungary 2000-2008 and molecular epidemiology of enterovirus 71, coxsackievirus A16, and echovirus 30. Virus Genes 2010;40:163-173.

22 Baek K, Yeo S, Lee B, Park K, Song J, Yu J, Rheem I, Kim J, Hwang S, Choi Y, Cheon D, Park J: Epidemics of enterovirus infection in Chungnam Korea, 2008 and 2009. Virol J 2011:13;8:297. 